\title{
Debridement in chronic osteomyelitis with benign osteopetrosis: A case report
}

\author{
YU-PING LIU ${ }^{1}$, XIANG-HUA LIN ${ }^{2}$, MAN-YUN YAN ${ }^{3}$, BAO-QUAN LIN $^{3}$ and MING-YING ZHUO ${ }^{3}$ \\ Departments of ${ }^{1}$ Endocrinology and Metabolism, ${ }^{2}$ Hematology and ${ }^{3}$ Otorhinolaryngology, \\ Xiamen Chang Gung Hospital, Xiamen, Fujian 361000, P.R. China
}

Received May 12, 2015; Accepted December 23, 2015

DOI: $10.3892 /$ etm.2016.3706

\begin{abstract}
Osteopetrosis is a rare bone disease caused by metabolic imbalances as a result of genetic mutations. For instance, autosomal dominant osteopetrosis is caused by a missense mutation of the $\mathrm{C} 1 \mathrm{CN} 7$ gene. This was first reported in 1904 and is thought to be caused by osteoclastic dysfunction and an impaired bone resorption ability. An accumulation of cortical bone mass during the remodeling of the medullary bone may increase the bone density and give rise to a hard marble consistency. Osteopetrosis can be divided into benign and malignant forms; however, no curative treatment exists for benign osteopetrosis. The management of complications, such as chronic osteomyelitis and fractures, serves a key role in influencing the patient survival rates. Previous studies have demonstrated that a combined treatment of hyperbaric oxygen (HBO) lavage for debridement of the necrotic region and high-dose systemic antibiotics may be effective in the management of osteopetrosis. The present study reported a case of chronic mandible osteomyelitis and fistula occurring in association with maxillary sinusitis, who was successfully treated by through nasal endoscopy, using repeated flushing and cleaning every 2 weeks as a form of debridement, in the absence of high-dose antibiotics and HBO.
\end{abstract}

\section{Introduction}

Osteopetrosis is a rare bone disease, with an estimated prevalence of 1 case in every 20,000 people (1). This disease, caused by metabolic imbalances resulting from genetic mutations, was first reported in 1904 by the German radiologist Heinrich Albers-Schönberg, as reviewed by Bollerslev (2) and García et al (3). Within one of a limited number of reports on this disease, García et al (3) demonstrated osteomyelitis of the

Correspondence to: Dr Ming-Ying Zhuo, Department of Otorhinolaryngology, Xiamen Chang Gung Hospital, 123 Xiafei Road, Xiamen, Fujian 361000, P.R. China

E-mail: zhuomy@adm.cgmh.com.cn

Key words: debridement, chronic osteomyelitis, benign osteopetrosis mandible in a patient with osteopetrosis. The pathophysiological mechanism of the disease involves an osteoclastic dysfunction that results in an impaired bone resorption ability; as a result, bone modeling and remodeling are inhibited. The cortical bone mass is therefore able to increase in density and develop into a hard, marble consistency when the medullary bone is remodelled. Two types of osteopetrosis exist: Malignant and benign, categorized based on mortality rate, and disease severity is highly heritable (1). Malignant osteopetrosis, or autosomal recessive osteopetrosis has a poor prognosis associated with fatality in infancy. Patients with ARO demonstrate a survival rate of $7 \%$, even subsequent to curative treatment such as allogenic hemopoietic stem cell transplantation (4). Currently, it is understood that autosomal dominant osteopetrosis (ADO) is a relatively benign disorder caused by a missense mutation in the ClCN7 gene (5). Benign osteopetrosis typically has a late onset in children and adults, the symptoms are milder and prognosis is better than in malignant osteopetrosis.

However, curative care is not available for patients with the benign form, thus only supportive care is provided. Osteopetrosis is difficult to treat and can cause complications, such as chronic osteomyelitis and bone fractures, as a result of poor blood supply and iatrogenic fractures. As there is no cure, symptom management is important in order to increase the patient survival rates.

The current study presents a case of chronic mandibular osteomyelitis and a maxillary sinusitis-generated fistula of benign osteopetrosis. The current recommended treatment options include high-dose systemic antibiotics, debridement of the necrotic region and hyperbaric oxygen (HBO) therapy (3). However, the current report describes treatment without the use of high-dose antibiotics or HBO therapy, a justified approach due to the insufficiency of the blood supply to the bone. Treatment of the debrided fistula, which included repeated flushing and cleaning using nasal endoscopy every 2 weeks in the acute infected period, was successful. Subsequently, a fistula developed through granuloma formation. Therefore, the present study investigated whether the debridement and drainage of pus from fistulas generated by maxillary sinusitis and chronic osteomyelitis is an effective treatment method.

\section{Case report}

In December 2014, a 50-year-old Chinese female was admitted to the Xiamen Chang Gung Hospital (Xiamen, China) for the 
A

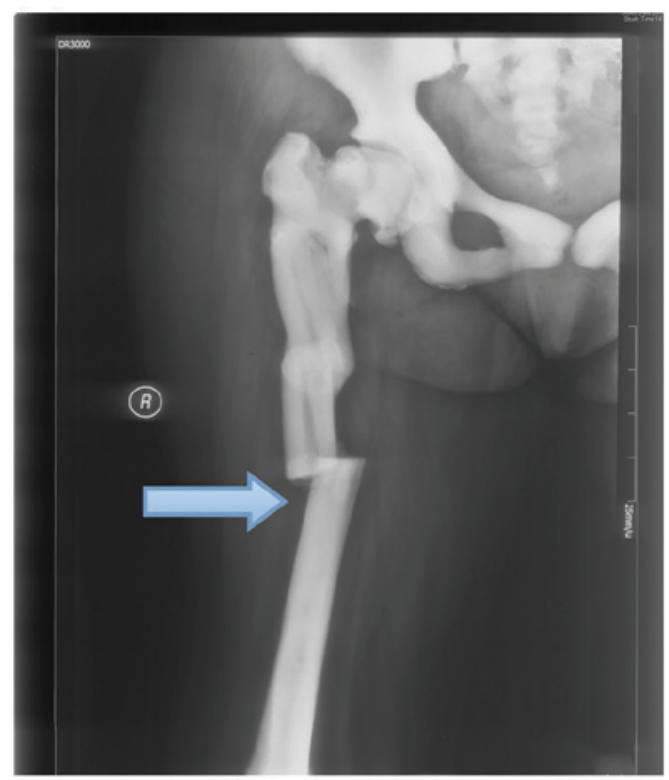

B

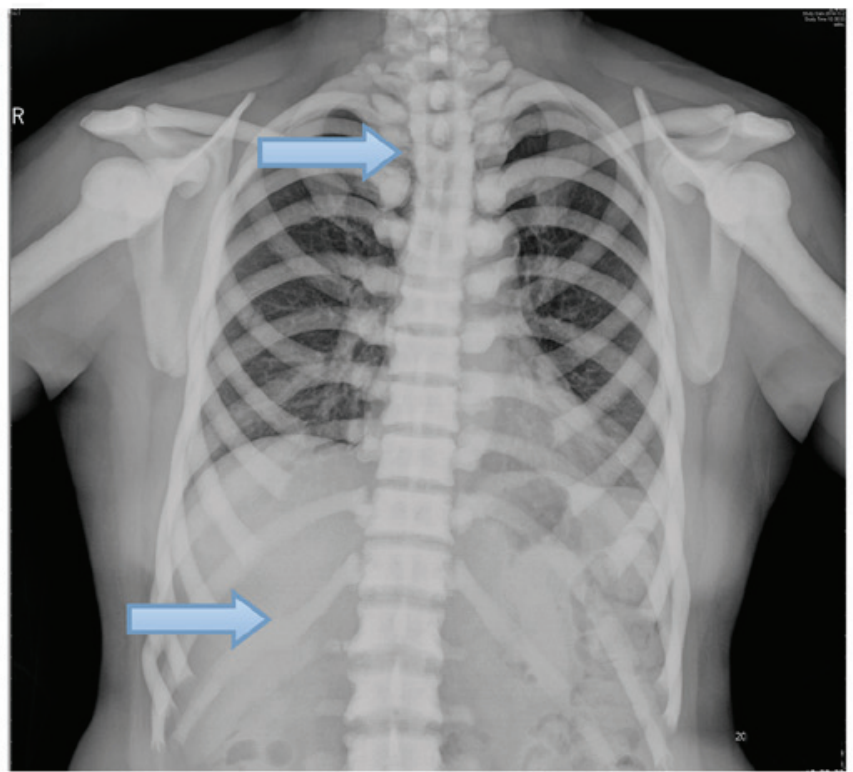

Figure 1. X-rays scans with blue arrows, identifying high bone density in (A) femoral and (B) rib and thoracic vertebra bones, resulting in fractures.

A

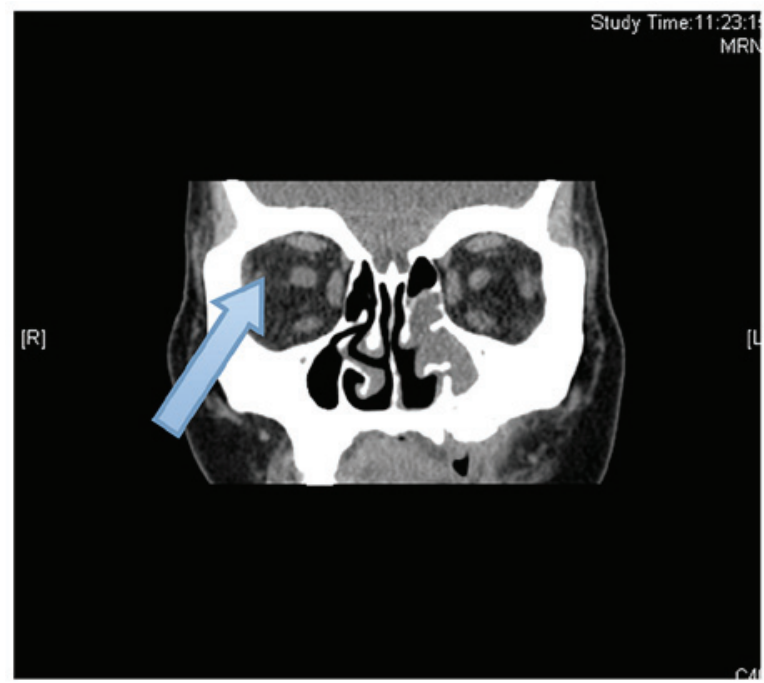

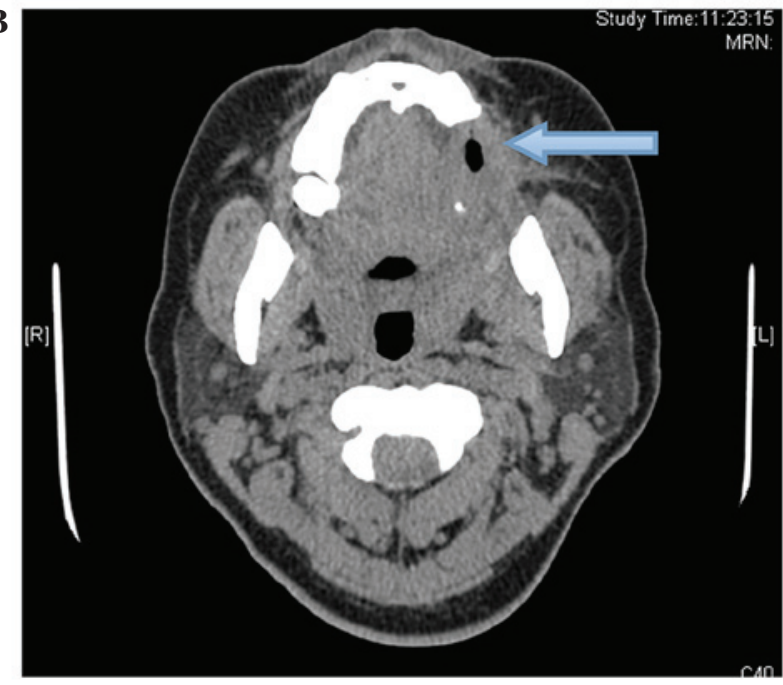

Figure 2. Non-contrast computed tomography scans identifying (A) evidence of low density bone in the posterior left side of the mandible (highlighted by the blue arrow) displaying destruction of the vestibular cortical, which is suggestive of chronic osteomyelitis with maxillary sinusitis, and (B) exposed sixth, seventh and eighth tooth agenesis in the upper left side and fistula formation (highlighted by the blue arrow).

evaluation and treatment of chronic purulent nasal discharge in the left side of the nose. The patient reported that the discharge appeared 3 years previously, following a tooth extraction from the left side of the mouth. Physical examination revealed red swelling in the left mandible. Intraoral examination revealed exposed necrotising bone in the left maxillary region, sixth, seventh and eighth tooth agenesis in the upper left side and fistula formation. The pus had accumulated in the mouth and the left side of the nose in the fistula, generated as a result of maxillary sinusitis. Previous medical history revealed that the patient was diagnosed with osteopetrosis at the age of 19 years old, with six rib, femoral and thoracic vertebra bone fractures (Fig. 1). The patient did not accept any treatment for the osteopetrosis, but was fitted with a plaster cast for 6 weeks. However, the patient developed splenomegaly due to secondary bone marrow suppression, subsequent to which the spleen was resected. Written informed consent was obtained from the patient for these and the following procedures. Examination of the patient's family history revealed that her older sibling was diagnosed with osteopetrosis at the age of 23 years old, but the patient's child had not developed the disease. Non-contrast computed tomography scans (GE Healthcare Life Sciences, Chalfont, UK) displayed evidence of low bone density in the posterior left side of the mandible with destruction of the vestibular cortex that was suggestive of a fistula, resulting from chronic osteomyelitis with maxillary sinusitis (Fig. 2). Subsequently, a bone marrow puncture, hematoxylin and eosin staining and brightfield imaging of these structures were performed. The medullary cavity was obliterated and the bone marrow was reported to be suppressed, determined 


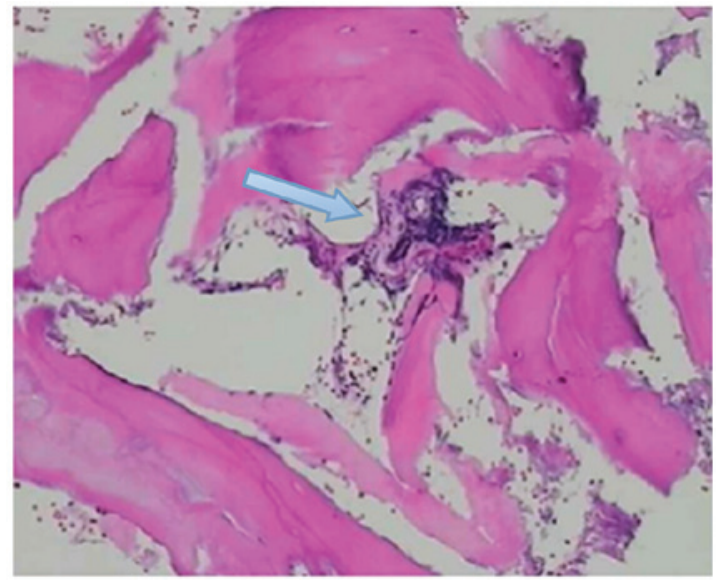

Figure 3. Suppressed bone marrow (identified by the blue arrow) scattered in hematopoietic tissue. Magnification, x400.

by hematoxylin and eosin staining using an Olympus BX51 brightfield microscope (Fig. 3). The sequestra were removed and debridement of the necrotic region was performed via nasal endoscopy, without antibiotics or any other form of treatment A month later, intravenous low-strength antibiotic therapy (1 g cefazolin, every 8 h; Mosinter Group, Ltd., Ningbo, China) was administered for a week, and the fistula was flushed and drained of pus via nasal endoscopy every 2 weeks. After a week, the infection had been successfully treated without the necessity of additional antibiotic treatment, chronic osteomyelitis was resolved and the fistula was replaced with fresh granulation after 2 months (Fig. 4).

\section{Discussion}

Osteopetrosis is a rare hereditary disease caused by an imbalance in bone metabolism, resulting from decreased osteoclast activity (6). This leads to a lack of bone remodeling, which can result in a high mortality rate in children within the first 10 years of their life (6). Early onset osteopetrosis is known as severe infantile malignant autosomal recessive disease, or intermediate mild autosomal recessive disease (6). This condition is typically fatal as a result of anemia with congestive heart failure or sepsis due to bone overgrowing in the bone marrow space. In adults, the same disease is known as benign ADO with a late onset, and has a lower mortality rate (3). At present, the curative treatment for malignant osteopetrosis is allogenic hemopoietic stem cell transplantation (4); however, only supportive care is available for benign osteopetrosis (7). Therefore, symptom management of benign osteopetrosis is important to increase the patient survival rates.

The present study reported the case of a patient diagnosed with benign osteopetrosis at the age of 19 years, with fractures identified based on presentation and radiology. In benign osteopetrosis, the most common complications are chronic osteomyelitis, occurring in $10 \%$ of cases involving the mandible (3), and bone fractures, in $78 \%$ of cases (8). The patient in the current study presented with chronic purulent nasal discharge in the left side of the nose, following tooth extraction from the left side of the mouth 3 years earlier associated with chronic osteomyelitis. Since the healing process in

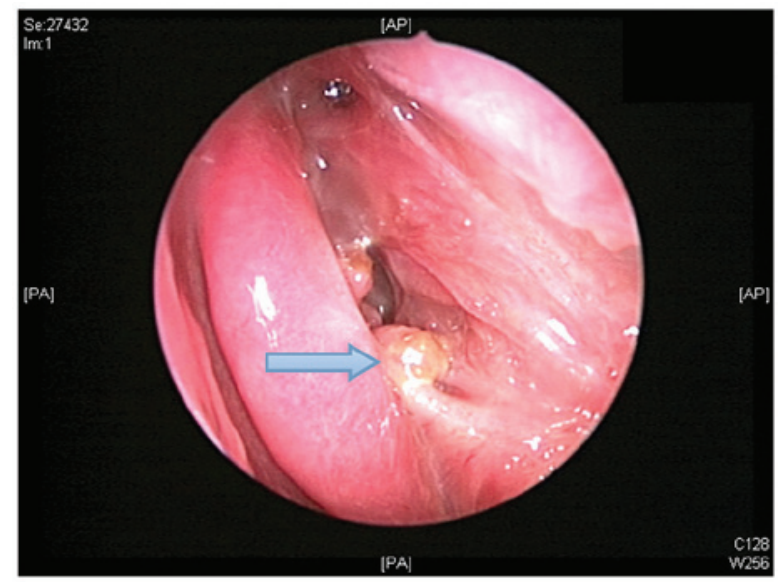

Figure 4. Nasal endoscopy revealing a fistula (identified by the blue arrow), which was healed with fresh granulation 1 month after debridement and drainage.

the bone is slow, it is difficult to fight and prevent infection in osteopetrosis (1). In ADO, care must be taken when removing the sequestra, since it is easy to create iatrogenic fractures due to dense bone, which are difficult to repair once the bone is fractured (9).

The current approaches of treating osteomyelitis in osteopetrosis are controversial. Currently, administering a high-dose of systemic antibiotics with debridement of the necrotic region alongside HBO therapy is recommended (3). However, as the patient in the current report presented with insufficient blood supply to the bone due to osteopetrosis, the use of high-dose antibiotics was not suitable. Instead, pus from the fistula was flushed and drained via nasal endoscopy every 2 weeks for 1 month, subsequent to careful removal of the sequestra and debridement of the necrotic region. Low dose and intensity antibiotics were administered following debridement, and the infection was cleared. To the best of our knowledge, this is the first case report indicating that debridement with low doses of antibiotics may be effective in the treatment of osteopetrosis.

Notably, patients with osteopetrosis should be aware of good dental care and oral hygiene. Decayed teeth should be endodontically treated and tooth extraction should be avoided, if possible, on the account of necrosis development. Currently, the debridement field does not have a clear boundary regarding infected bones. Care must therefore be taken when removing the necrotic bone to prevent iatrogenic fractures, which may occur due to inexperience of the surgeon (9). The findings of the present study demonstrated that debridement and drainage of pus from fistulas are important, and possibly a more effective strategy compared with high-dose antibiotic usage alone in osteopetrosis.

In conclusion, the complications of osteopetrosis include bone fractures and chronic osteomyelitis, which are difficult to treat. In a patient with osteopetrosis, tooth extraction should be avoided to prevent these complications. Currently, there is no cure for osteopetrosis and, therefore, symptom management is important to increase the patient survival rates. In the present study, it was demonstrated that debridement and drainage of pus from fistulas may be more effective than high-dose antibiotic usage in treating osteopetrosis. 


\section{References}

1. Smith NH: Albers-Schönberg disease (osteopetrosis). Report of a case and review of the literature. Oral Surg Oral Med Oral Pathol 22: 699-710, 1966

2. Bollerslev J: Autosomal dominant osteopetrosis: Bone metabolism and epidemiological, clinical, and hormonal aspects. Endocr Rev 10: 45-67, 1989.

3. García CM, García MA, García RG and Gil FM: Osteomyelitis of the mandible in a patient with osteopetrosis. Case report and review of the literature. J Maxillofac Oral Surg 12: 94-99, 2013.

4. Juggins KJ, Walton GM and Patel M: Osteomyelitis complicating osteopetrosis - a case report. Dent Update 28: 509-511, 2001 .

5. Battaglia MA, Drigo P, Laverda AM, Antolini A, Venuleo M, Miotti A and Pavone L: Juvenile osteomyelitis and osteopetrosis. A case report. Minerva Stomatol 40: 125-127, 1991 (In Italian).

6. Bollerslev J and Andersen PE Jr: Radiological, biochemical and hereditary evidence of two types of autosomal dominant osteopetrosis. Bone 9: 7-13, 1988.

7. Coudert AE, de Vernejoul MC, Muraca M and Del Fattore A: Osteopetrosis and its relevance for the discovery of new functions associated with the skeleton. Int J Endocrinol: 372156, 2015.

8. Bénichou OD, Laredo JD and de Vernejoul MC: Type II autosomal dominant osteopetrosis (Albers-Schönberg disease): Clinical and radiological manifestations in 42 patients.Bone 26: 87-93, 2000.

9. van Hove RP, de Jong T and Nolte PA: Autosomal dominant type I osteopetrosis is related with iatrogenic fractures in arthroplasty. Clin Orthop Surg 6: 484-488, 2014. 